\title{
Impact of imagery rescripting on adverse self- defining memories and post-recall working selves in a non-clinical sample: a pilot study
}

\section{Soljana Çili, Sharon Pettit \& Lusia Stopa}

To cite this article: Soljana Çili, Sharon Pettit \& Lusia Stopa (2016): Impact of imagery rescripting on adverse self-defining memories and post-recall working selves in a non-clinical sample: a pilot study, Cognitive Behaviour Therapy, DOI: 10.1080/16506073.2016.1212396

To link to this article: http://dx.doi.org/10.1080/16506073.2016.1212396

电 Published online: 29 Jul 2016.

Submit your article to this journal $\pi$

Џll Article views: 34

Q View related articles $\llbracket$

View Crossmark data $\asymp$ 


\title{
Impact of imagery rescripting on adverse self-defining memories and post-recall working selves in a non-clinical sample: a pilot study
}

\author{
Soljana Çili (D), Sharon Pettit and Lusia Stopa \\ Psychology Academic Unit, University of Southampton Highfield, Southampton, UK
}

\begin{abstract}
Imagery rescripting (ImRS) effectively targets intrusive images and symptoms in a number of disorders, but the mechanisms of change behind it are not yet clear. This study investigated the impact of ImRS on the characteristics of adverse self-defining memories and postrecall working selves in a non-clinical sample. In the first session, participants recalled an adverse memory and completed state self and affect measures. Then they attended an ImRS session and a followup session one week later. Participants rated their memory as less negative, less distressing and less important for their sense of self at follow-up compared to the first session. They also reported higher state self-esteem and positive affect, as well as reduced negative affect and anxiety after recalling the memory. Results suggest that, by modifying the meaning of adverse memories, ImRS may facilitate their integration with individuals' sense of self and reduce the negative impact that they have on individuals' online representation of the self when retrieved. An implication of these findings is that cognitivebehavioural therapy may need to conceptualise the self in broader terms, moving beyond core beliefs. To complement this, research could focus on changes in patients' sense of self in order to understand the mechanisms through which interventions like ImRS work.
\end{abstract}

\section{ARTICLE HISTORY}

Received 6 February 2016 Accepted 10 July 2016

\section{KEYWORDS}

Imagery; imagery rescripting; self; self-defining memories; trauma

Imagery rescripting (ImRS) is a cognitive-behavioural technique which aims to modify the meanings associated with negative or traumatic memories (Arntz \& Weertman, 1999; Smucker, Dancu, Foa, \& Niederee, 1995). Intrusive images of these memories are often associated with negative beliefs such as I am a failure (e.g. Wells \& Hackmann, 1993), negative emotions such as anxiety and helplessness (e.g. Speckens, Hackmann, Ehlers, \& Cuthbert, 2007), and dysfunctional behaviours such as self-induced vomiting in bulimia nervosa (Hinrichsen, Morrison, Waller, \& Schmidt, 2007). ImRS has been found to reduce image frequency and vividness and to alleviate symptoms in diverse disorders, including depression (Brewin et al., 2009), social phobia (Lee \& Kwon, 2013; Wild, Hackmann, \& Clark, 2007, 2008), and posttraumatic stress disorder (e.g. Arntz, Sofi, \& Van Breukelen, 2013; Grunert, Smucker, Weis, \& Rusch, 2003). It can also reduce the valence of the associated 
memory (Dibbets, Poort, \& Arntz, 2012) and the strength of the encapsulated negative beliefs (e.g. Lee \& Kwon, 2013; Wild et al., 2007, 2008). Despite this promising evidence, the mechanisms of change in ImRS are not yet clear.

Several explanations have been proposed with regard to the changes promoted by ImRS (see Dibbets \& Arntz, 2016; Reimer \& Moscovitch, 2015). Arntz (Arntz, 2011; Arntz \& Weertman, 1999), for example, suggests that ImRS might work by modifying the meaning of the adverse experience and, consequently, the content of related maladaptive schemas (the unconditioned stimulus representation). As a result, conditioned stimuli (e.g. trauma reminders) trigger different conditioned responses (e.g. emotions) that reflect the new meanings. Brewin (2006), on the other hand, questions the idea that cognitive-behavioural interventions modify negative schemas and argues that they reduce the accessibility of such schemas. He suggests that individuals possess multiple schemas or self-representations that compete for retrieval. Which self-representations are active at any one time is determined by factors such as environmental triggers and the salience of the self-representations. According to this theory, ImRS may add contextual information to the predominantly sensory representations of the adverse memory and make the new representations more likely to win the retrieval competition over the original representations (see Brewin, Gregory, Lipton, \& Burgess, 2010). Other explanations behind the changes involved in ImRS include ImRS facilitating the elaboration and integration of the adverse memory within the individual's autobiographical knowledge base and life story and allowing the expression of responses (e.g. emotions) that were activated but not expressed during the adverse experience (see Dibbets \& Arntz, 2016).

In our view, Brewin's (2006) hypothesis offers a plausible explanation of how ImRS might work. Although to date no studies have tested it, there is some indirect support for it. Individuals report different self-aspects (state self-esteem, self-cognitions, goals) after recalling self-defining memories (SDMs: highly vivid, emotional and accessible memories that have shaped their sense of self; Singer \& Salovey, 1993), depending on the memories' valence and the extent to which individuals have drawn abstract meaning from them (Çili \& Stopa, 2015). Specifically, the recall of positive SDMs is associated with higher post-recall state self-esteem, a marginally higher proportion of goals related to recreation/exploration activities, and a marginally lower proportion of achievement-related goals than the recall of negative SDMs. The recall of SDMs from which meaning has not been abstracted is associated with the reporting of a higher proportion of emotion-related self-cognitions (e.g. scared) than the recall of memories from which individuals have abstracted meaning, potentially indicating a more intense emotional response. Furthermore, following ImRS some patients experience spontaneous behavioural changes (e.g. increased assertiveness) that may be due to an increased accessibility of positive self-representations and the activation of previously learnt behaviours (Brewin et al., 2009). The findings of Çili and Stopa (2015) and Brewin et al. (2009) are consistent with the hypothesis that individuals have different self-representations which are activated in response to memory recall and whose accessibility may be modified as a result of ImRS.

Linked to Brewin's (2006) hypothesis is the self-memory system (SMS) model (Conway, 2005; Conway \& Pleydell-Pearce, 2000; Conway, Singer, \& Tagini, 2004), which argues that individuals possess a stable long-term self and multiple working selves that guide cognition, affect and behaviour. According to the model, the SMS enables individuals to maintain a coherent sense of self while adapting to their circumstances. The long-term self contains the individual's autobiographical memories and conceptual information about 
the self that is organised in the form of self-structures (e.g. schemas, possible selves, beliefs and values). A subset of these structures, the working self, is activated in response to shifts in environmental demands. The working self that is active at any one time consists of goals and self-images representing mental models of the self (i.e. aspects of the conceptual self) and helps individuals respond adequately to environmental demands. A change in these demands is associated with a change in goal status. In response to this change, the long-term self searches the individual's autobiographical memory for a past SDM and working selves that may be relevant to the current situation. In the end, this search yields a specific SDM, an affective response, and a related working self that guides the individual's response to the goal status change (Conway et al., 2004). According to this model, working selves associated with adverse memories are highly salient and accessible because these memories threaten the achievement of the individual's goals and are not integrated within the autobiographical knowledge of the long-term self (Conway, Meares, \& Standart, 2004). The literature is not clear with regard to the similarities and differences between working selves and schemas or self-representations. We have chosen to use Conway's (e.g. 2005) terminology of the working self because the construct is clearly defined within the SMS model (and therefore easier to investigate), incorporates knowledge about the self as well as autobiographical experience, and accords well with patients' descriptions of changes to sense of self as a result of traumatic experience (I feel as if my old self has died, I can't be the person I was anymore since the accident). However, we acknowledge that there is considerable overlap between the conceptualisation of the working self and the concept of schemas, and in particular the notion of "schema mode" (Young, 1999; Young, Klosko, \& Weishaar, 2003).

Based on the SMS model and Çili and Stopa's (2015) findings, we propose that intrusive images are components of working selves related to adverse SDMs and that the affective and behavioural responses associated with them are a result of the activation of these working selves. We suggest that by changing the meaning and the valence of adverse memories, ImRS may modify the impact that the retrieval of these memories has on working self activation. In line with the retrieval competition account, we hypothesise that ImRS may reduce the accessibility of negative memory-related working selves and increase the accessibility of positive ones. Specifically, we hypothesise that following rescripting, the retrieval of an adverse memory will be associated with the activation of a more positive working self (consisting of positive self-images, goals, and affect) compared to the retrieval of the same memory before rescripting.

Any attempts to test this hypothesis face the challenge of operationalising the working self. As stated earlier, in the SMS model (e.g. Conway, 2005) the working self is described as consisting primarily of self-images and goals. In an attempt to capture these components, in a previous study (Çili \& Stopa, 2015) we asked participants to complete the TwentyStatement Test (Kuhn \& McPartland, 1954) and a measure of personal goals (Emmons, 1986; Sutherland \& Bryant, 2005) after recalling a positive or a negative SDM. Specifically, we instructed them to provide up to 20 answers to the question "Who am I?" and to list up to 15 goals that were important for them to achieve. We then coded the self-cognitions and the goals they provided. Results suggested that these two measures may not be sensitive enough to detect the activation of a different working self at the time of data collection. Furthermore, it may be difficult for individuals to verbalise and distinguish between the fleeting self-images and goals that are active at any one time. However, our results suggested that measures of state self-esteem, state self-concept clarity, and state affect can function 
as proxies for the working self. Self-esteem is the individual's evaluation of the content of his/her self-concept, whereas self-concept clarity refers to the stability of this content and the individual's certainty of it. The activation and prominence of certain self-images can lead to changes in the individual's current self-evaluation that can be measured by state self-esteem questionnaires. These changes may in turn influence the individual's current certainty about the stability of the self-concept or the degree of integration between different working selves and the long-term self, leading to fluctuations in self-concept clarity. At the same time, these changes in view of self may be accompanied by affective changes related to the individual's subjective experience of the self as more or less positive or negative. Based on this reasoning and on our previous findings, in this study we decided to assess state self-esteem, self-concept clarity, and affect following memory recall in order to understand the post-recall working self.

Self-esteem and self-concept clarity are positively related to each other and to psychological well-being (Campbell et al., 1996; Constantino, Wilson, Horowitz, \& Pinel, 2006; Paradise \& Kernis, 2002). Another construct which is related to self-esteem, self-concept clarity, and psychological well-being — and which we also used to understand the working self-is the structure or organisation of the self-concept (Showers, 1992; Showers, Ditzfeld, \& Zeigler-Hill, 2015; Zeigler-Hill \& Showers, 2007). Self-structure may be compartmentalised (positive and negative self-beliefs are organised into different self-aspects or self-representations) or integrative (self-aspects contain both positive and negative beliefs). Depending on which self-aspects are important/salient, the self-structure may be positive or negative. It may influence how individuals respond to environmental stimuli, with compartmentalised individuals experiencing fluctuating self-esteem and self-concept clarity as they access positive or negative self-representations and integrative individuals being more stable as they access self-representations containing both positive and negative information (Showers et al., 2015; Zeigler-Hill \& Showers, 2007). Self-structure, however, may change as a result of experiences such as trauma or therapy (Showers, Limke, \& Zeigler-Hill, 2004). Showers et al. (2004) argue that cognitive-behavioural interventions promote the development of integrative self-structures or positively compartmentalised ones because they encourage patients to acknowledge positive attributes and/or link them to negative ones. In the light of this theory and of the evidence on the role of self-structure in self-representation activation, we hypothesised that ImRS may lead to a change in self-structure which in turn influences the types of self-representations or working selves that are activated in response to environmental triggers.

This study aimed to investigate some of the basic ImRS cognitive changes in a non-clinical sample in a preliminary attempt to contribute to the debate about potential mechanisms of change in ImRS. The study consisted of three sessions. In the first session, participants recalled an adverse, significantly disturbing, and intrusive SDM. We assessed memory characteristics, self-structure, and the post-recall working self (state self-esteem, self-concept clarity, self-description consistency, and affect). The memory was rescripted in the second session. In the third session, participants recalled the memory again and completed the self and memory measures. We predicted that, following ImRS, participants would report: (1) reduced memory vividness, memory distress, and encapsulated belief strength; (2) higher state self-esteem, state self-concept clarity, and self-description consistency; and (3) greater positive affect and reduced negative affect. Because to date there is no empirical evidence 
on self-structure changes following treatment, we had no specific hypotheses about this variable and included it for exploratory purposes.

\section{Method}

\section{Participants}

The study was advertised to individuals at a local university who were troubled by negative memories and related images. Thirty-nine individuals completed an online questionnaire that assessed their exposure to adverse experiences. Ten were excluded because they reported severe or multiple traumas (e.g. sexual assault and physical and/or emotional abuse) and might find ImRS too distressing or require long-term treatment. Five declined to participate. Three participants completed only Session 1: one withdrew for unknown reasons, one withdrew after experiencing an increase in the frequency of intrusive images, and for one participant the clinicians decided that ImRS would be inappropriate given the brief period of time that had elapsed since the occurrence of the trauma. Another participant withdrew after it emerged in Session 2 that she had already come to terms with her experience. The individuals who were excluded or withdrew from the study were informed of services providing psychological support.

Twenty participants (19 females, 1 male) completed the study in return for course credits or payment. Their ages ranged from 18 to 41 years $(M=22.05$ years, $\mathrm{SD}=5.59)$. Four participants had received treatment for psychological problems (depression, stress/anxiety, eating disorder) in the past, but none was currently in treatment. In the screening questionnaire, participants reported between 1 and 6 of the negative events listed $(M=3.30, \mathrm{SD}=1.42)$. The most frequently reported events were injury, illness or death of a family member $(n=17)$; personal injury or illness $(n=12)$; and emotional abuse $(n=11)$.

\section{Imagery interview}

In Session 1, participants completed a semi-structured interview adapted from Hackmann, Clark, and McManus (2000). Using instructions adapted from Beike and Wirth-Beaumont (2005) and Jobson and O'Kearney (2008), they were first asked to recall a negative SDM that was associated with strong emotions and vivid images, that had happened at a specific time and place, that still troubled them, and that had influenced the way they saw themselves. When they had identified a memory that met these criteria, they were asked to describe it in one or two sentences and to indicate how old they were at the time. They were then asked to indicate how negative the experience was and to what extent it had influenced the way they saw themselves on a scale from 0 (not at all) to 10 (extremely). If the valence and/or influence rating was below 5, participants were asked to think of another experience. The aim was to ensure that the memories were sufficiently negative and self-relevant.

Once they had identified a memory that met the inclusion criteria, participants were asked to close their eyes, focus on the memory in order to make it as vivid as possible in their mind, and describe it in the present tense. While describing the memory, they rated its characteristics and reported the emotions, thoughts, and physical sensations associated with it. The researcher elicited the meaning of the memory with the questions In what ways do you think this experience has influenced you? What does the memory say about you as a 
person? Does it say anything about other people/the world in general? Participants summarised this meaning in one statement and rated their belief in it. The interview lasted about $30 \mathrm{~min}$ and was audio recorded. It was repeated in Session 3.

\section{Measures}

\section{Questionnaire on adverse experiences}

This questionnaire was adapted from the Posttraumatic Diagnostic Scale (Foa, Cashman, Jaycox, \& Perry, 1997) and the Stressful Life Events Screening Questionnaire (Goodman, Corcoran, Turner, Yuan, \& Green, 1998). Participants indicated which of 15 negative events (e.g. personal injury or illness, physical or emotional abuse, sexual assault) they had experienced.

\section{Memory characteristics}

In Sessions 1 and 3, participants rated on a 0 (not at all) to 10 (extremely) scale the valence of their experience, the extent to which it had influenced how they saw themselves, its vividness, and the distress associated with it when it happened and when they recalled it in the session.

\section{Encapsulated belief strength}

In Sessions 1 and 3, participants rated how much they believed the statement that summarised the meaning of the memory on a scale from 0 (not at all) to 10 (extremely).

\section{State self-esteem scale (SSES; McFarland \& Ross, 1982)}

The SSES consists of 12 of McFarland and Ross's (1982) self-esteem factors. Participants rated how they felt "right now" with regard to items such as inadequate and smart on a scale from 1 (not at all) to 11 (extremely). The scores to individual items were summed after the negative items were reverse scored. Total scores in this questionnaire range from 12 to 132 , with high scores indicating high state self-esteem. Cronbach's $\alpha$ in this study varied from .90 to .95 .

\section{State self-concept clarity scale (SSCCS; Nezlek \& Plesko, 2001)}

The 4 items constituting this scale are taken from Campbell et al's (1996) self-concept clarity scale. Participants indicated how much items such as My beliefs about myself seem to change very frequently applied to them "right now" on a scale from 1 (strongly disagree) to 5 (strongly agree). All the items were reverse scored and individual scores were summed. Total scores in this questionnaire range from 4 to 20 and high scores indicate high state self-concept clarity. In our sample, Cronbach's $\alpha$ varied from .83 to .93 .

\section{Positive and negative affect scales (PANAS; Watson, Clark, \& Tellegen, 1988)}

This questionnaire consists of two 10-item subscales. Participants rated the extent to which they were experiencing positive and negative emotions "right now" on a scale from 1 (very slightly or not at all) to 5 (extremely). Total scores, which may range from 10 to 50, were obtained separately for positive and negative affect. Cronbach's $\alpha$ for the subscales varied from .81 to .95 . 


\section{State subscale of the state-trait anxiety inventory (STAI-S; Spielberger, Gorsuch, Lushene, Vagg, \& Jacobs, 1983)}

The 20 items of this scale measure the intensity of the anxiety experienced at a particular moment. Participants rated how they felt "right now" on a scale from 1 (not at all) to 4 (very much so). Overall scores range from 20 to 80, with higher scores indicating higher levels of anxiety. Cronbach's $\alpha$ varied from .93 to .95 .

\section{Me/not me task (Markus, 1977)}

This computer task assessed the consistency of participants' self-description (an aspect of self-concept clarity). It involved 10 practice trials and 50 experimental trials. The experimental trials included pairs of opposite adjectives such as interesting-boring. Positive and negative adjectives did not differ in terms of absolute valence, $t(48)=0.95, p=.35$. Adjectives appeared at the centre of a computer screen in a randomised order. Participants pressed $Y$ or $N$ to indicate whether the adjectives described them or not. The adjective remained on the screen until participants responded or $8 \mathrm{~s}$ had elapsed and was followed by an asterisk that appeared at the centre of the screen for $1 \mathrm{~s}$. Participants then indicated how confident they were about their answer on a scale from 1 (not at all) to 7 (extremely). Response times were recorded.

This task yields three measures of self-concept clarity: consistency, confidence ratings, and reaction times. For the purposes of this study, we focused only on consistency. Participants are said to be consistent when they respond yes to one adjective and no to its opposite. Consistent responses are given a score of 1 and inconsistent responses a score of 0 .

\section{Card-sorting task (Showers, 1992, adapted from Linville, 1987)}

This task assesses self-structure. Participants were given 40 cards containing 20 positive and 20 negative adjectives (e.g. popular, incompetent) that did not differ significantly in terms of absolute valence, $U=148.00, p=.16$. Participants sorted the cards into groups that described meaningful aspects of themselves or their life, using as many cards as necessary in each group. Next, they indicated how positive, negative and important each self-aspect was on a scale from 1 (not at all) to 7 (very). The data were used to generate three indices:

(1) $\Phi$, which indicates self-structure and may vary from 0 (perfect integration) to 1 (perfect compartmentalisation);

(2) Differential Importance (DI), which indicates the importance of the self-aspects and may range from -1 (negative self-aspects rated more important) to +1 (positive self-aspects rated more important); and

(3) Neg, which represents the proportion of negative attributes present in the card sort.

\section{Intervention}

The ImRS intervention was based on Arntz and Weertman's (1999) protocol and was delivered by two clinical psychologists who had extensive experience in using ImRS and other imagery techniques. The protocol consisted of three stages. In Stage 1, participants relived their negative experience. In Stage 2, they relived it from the perspective of their current self and intervened in the memory. Interventions consisted mainly of the current 
self introducing elements that changed the way the event unfolded and made it more positive for their younger self (i.e. their self at the time the event occurred). One participant who discovered that her partner had been lying to her about committing a crime and was arrested, for example, reassured the younger self that what happened was not her fault and that she was not stupid for believing him. She also brought in her father, who comforted and helped her understand what had happened by obtaining more information about the arrest. In Stage 3, participants took the perspective of their younger self and asked the current self for any help or emotional reassurance that was required. The participant who learnt about her partner's arrest, for example, imagined her father holding her hand and taking her out for fresh air. ImRS lasted about $50 \mathrm{~min}$ and was video recorded.

We assessed adherence to the protocol using a scale developed for the purposes of this study. The first part of the scale assessed whether the clinicians followed the steps outlined in the protocol. The second part assessed how skillfully they delivered the session (e.g. responded to participants' distress) and used a rating scale from 0 (not at all) to 100 (extremely). The first author assessed adherence in all sessions. An independent clinician with experience in imagery work assessed $20 \%$ of the sessions. Inter-rater reliability for adherence was adequate (Cohen's $\kappa=.74$ ), so the first author's ratings were used in the analysis. Therapists completed $94 \%$ of the steps prescribed in the protocol and administered the intervention skillfully $(M=92.63, \mathrm{SD}=3.85)$. There were no significant differences between them in terms of protocol adherence $(U=44.50, p=.66)$ or skill in conducting the session $(t(18)=1.33, p=.20)$.

\section{Procedure}

Participants attended three sessions held approximately 1 week apart. At the beginning of Session 1, they provided demographic information. Next, they completed the interview, followed by the self and affect questionnaires. Finally, they completed the me/not me and the card-sorting tasks. In Session 2, participants rescripted their memory and completed the self and affect questionnaires. The me/not me and the card-sorting tasks were not administered in this session to avoid making excessive demands on the participants after the potentially intense rescripting. The structure of Session 3 was similar to that of Session 1. At the end of the study, participants were fully debriefed.

\section{Results}

\section{Memory characteristics}

Experiences recalled by participants included the death of a family member or friend, finding out about parents' divorce, having major life-changing surgery, and serious conflicts with close friends or abusive partners. The age at which these experiences had occurred varied from 7 to 30 years $(M=17.73$ years, $\mathrm{SD}=5.07)$. All participants reported that their memory had a visual component. Eighteen participants (90\%) reported that it had an auditory component (e.g. people's voices). Nineteen participants (95\%) said that it included bodily sensations such as sweating and breathing difficulties. For five participants (25\%) the memory included at least one particular smell (e.g. hospital smell) and for two participants $(10 \%)$ it included a particular taste (e.g. anaesthetic). The most frequently reported emotions 
Table 1. Memory characteristics in Sessions 1 and 3.

\begin{tabular}{|c|c|c|c|c|c|c|c|}
\hline \multirow[b]{2}{*}{ Variable } & \multicolumn{2}{|c|}{ Session 1} & \multicolumn{2}{|c|}{ Session 3} & \multirow[b]{2}{*}{$F(1,19)$} & \multirow[b]{2}{*}{$p$} & \multirow[b]{2}{*}{ Cohen's $d$} \\
\hline & M & SD & M & SD & & & \\
\hline Memory valence & 8.88 & 1.10 & 8.23 & 1.32 & 6.05 & .02 & -0.54 \\
\hline Memory influence & 7.34 & 1.43 & 6.38 & 1.42 & 6.11 & .02 & -0.67 \\
\hline Image vividness & 7.68 & 0.98 & 7.55 & 1.20 & 0.13 & .73 & -0.12 \\
\hline Post-event distress & 9.13 & 0.86 & 9.01 & 0.80 & 0.43 & .52 & -0.14 \\
\hline Post-recall distress & 5.80 & 1.58 & 4.07 & 2.24 & 14.88 & $<.01$ & -0.91 \\
\hline Belief strength & 7.40 & 2.64 & 5.80 & 2.75 & 13.36 & $<.01$ & -0.59 \\
\hline
\end{tabular}

Table 2. Descriptive statistics for self and affect measures in Sessions 1, 2, and 3.

\begin{tabular}{lcccccc}
\hline & \multicolumn{2}{c}{ Session 1 } & \multicolumn{2}{c}{ Session 2 } & \multicolumn{2}{c}{ Session 3 } \\
\cline { 2 - 7 } Variable & $M$ & SD & $M$ & SD & $M$ & SD \\
\hline State self-esteem & 81.15 & 26.82 & 93.15 & 18.43 & 94.45 & 19.96 \\
State self-concept clarity & 13.73 & 3.76 & 14.43 & 3.95 & 14.70 & 4.26 \\
Me/not me consistency $^{\mathrm{a}}$ & 20.35 & 2.85 & - & - & 20.25 & 3.39 \\
$\Phi^{\mathrm{a}}$ & .39 & .14 & - & - & .38 & .18 \\
$\mathrm{Dl}^{\mathrm{a}}$ & .31 & .36 & - & - & .44 & .47 \\
Neg $^{\text {a }}$ & .23 & .11 & - & - & .21 & .13 \\
Positive affect & 23.15 & 8.15 & 26.11 & 8.35 & 28.25 & 9.88 \\
Negative affect & 18.75 & 7.28 & 16.16 & 5.09 & 13.65 & 4.18 \\
State anxiety & 45.30 & 10.63 & 40.20 & 12.92 & 36.00 & 12.59 \\
\hline
\end{tabular}

Notes. $\Phi=$ index of self-structure; $\mathrm{DI}=$ differential importance in the card-sorting task; Neg = proportion of negative attributes present in card sort.

aThe me/not me task and the card-sorting task were not administered in Session 2.

were anger $(n=8)$, shock $(n=6)$, confusion $(n=6)$, helplessness $(n=6)$, fear $(n=5)$ and sadness $(n=5)$. The encapsulated beliefs included I am helpless/a failure, You cannot trust people, and Anything can happen and everything can go in the split of a second.

Table 1 presents participants' memory ratings. A repeated-measures multivariate analysis of variance (MANOVA) with session as within-subjects factor revealed that there was a significant multivariate difference between Session 1 and Session 3 ratings, $F(6,14)=3.02$, $p=.04$, Wilks' $\lambda=.44$. Participants rated their memory as less negative and less important for their self-view in Session 3. In addition, they were significantly less distressed after recalling the memory and believed less strongly in the encapsulated belief. Participants' perception of how distressing the event was when it happened and the vividness of the memory-related images did not change significantly.

\section{Self and affect measures}

Table 2 presents participants' scores on self and affect measures completed after memory recall in Sessions 1 and 3 and after ImRS in Session 2. There was a significant multivariate difference between the sessions in terms of the self measures, $F(4,74)=3.52, p=.01$, Wilks' $\lambda=.71$. The effect of time was significant for state self-esteem $(F(2,38)=5.64, p=.01)$, but not for state self-concept clarity $(F(2,38)=1.71, p=.20)$. Pairwise comparisons revealed that state self-esteem increased significantly from Session 1 to Session $2(p=.04)$ and from Session 1 to Session $3(p=.01)$. State self-concept clarity did not change significantly between sessions (all ps > .16). Cohen's $d$, calculated taking into account Session 1 and Session 3 scores, was 0.57 for state self-esteem and 0.24 for state self-concept clarity. 
The multivariate difference between the sessions was not significant for affect, $F(6,68)=$ $1.91, p=.09$, Wilks' $\lambda=.73$. However, there was a significant effect of time on positive affect $(F(2,36)=3.68, p=.04)$, negative affect $(F(2,36)=4.35, p=.02)$, and anxiety $(F(2,36)=$ $4.77, p=.01)$. Pairwise comparisons showed that state anxiety decreased and positive affect increased significantly from Session 1 to Session 3 ( $p=.01$ for both variables). Negative affect decreased significantly from Session 2 to Session $3(p=.03)$ and from Session 1 to Session $3(p=.02)$. Cohen's $d$ was 0.57 for positive affect, -0.89 for negative affect, and -0.80 for state anxiety.

Because participants' self-description consistency and self-structure were measured only in Sessions 1 and 3, they were analysed separately. There was no significant change in self-consistency following ImRS, $t(19)=0.15, p=.89$, Cohen's $d=-0.03$. Three participants were excluded from the card-sorting task data analysis: one for failing to follow instructions and two for reporting a perfectly compartmentalised self-organisation either in Session 1 or in Session 3 (see Showers, 1992). The remaining 17 participants generated a mean of 5.94 self-aspects in Session $1(\mathrm{SD}=1.78)$ and 6.06 self-aspects in Session $3(\mathrm{SD}=1.71)$. Each self-aspect contained on average 12.61 adjectives $(S D=3.62)$ in Session 1 and 12.94 adjectives $(\mathrm{SD}=3.14)$ in Session 3. There were no significant differences in the number of self-aspects generated $(t(16)=-.29, p=.78)$ or the attributes in each self-aspect $(t(16)=-.58$, $p=.57)$ between testings. Participants displayed an integrative self-structure $(\Phi)$, considered the positive self-aspects more important than the negative ones (DI), and reported a low proportion of negative attributes (Neg). A repeated-measures MANOVA revealed that there was no significant multivariate difference between sessions in $\Phi$, DI, and Neg values, $F(3$, $14)=1.44, p=.27$, Wilks' $\lambda=.76$. None of these values changed significantly from Session 1 to Session 3 (all ps > .17). Cohen's $d$ was -0.06 for $\Phi, 0.31$ for DI, and -0.17 for Neg.

\section{Discussion}

This study aimed to understand some of the basic cognitive changes involved in ImRS. Our findings are consistent with previous research showing that ImRS reduces the strength of the encapsulated belief and makes memory-related images less distressing (e.g. Brewin et al., 2009; Wild et al., 2007). They add to existing evidence by showing that participants perceived their experience as less negative and less influential for their self-views following ImRS. This suggests that ImRS helped participants to update the meaning they had attached to the memory. Anecdotal evidence supports this proposition. Several participants commented that ImRS helped them to see how much they had matured since their negative experience had occurred. Prior to the rescript, some of them had avoided thinking about the memory and this avoidance may have blocked the processing needed to spontaneously update it. ImRS may have helped them to access information about how events had really unfolded and to question negative beliefs such as I am stupid.

The fact that participants repeatedly revisited their SDM during the study may explain why the memory-related images remained vivid after ImRS. Perhaps vividness reduction may occur at a later stage and require a longer follow-up. Alternatively, it may not occur. In fact, not all previous ImRS studies have found a reduction in image vividness (e.g. Nilsson, Lundh, \& Viborg, 2012). Wild et al. (2007) found such a reduction, but it was not maintained at 1 -week follow-up. This evidence suggests that vividness reduction may not be essential 
for the other changes promoted by ImRS. McKay, Singer and Conway (in press) actually argue that a healthy autobiographical memory system contains vivid memories.

If the above explanation is correct, the changes in memory distress and encapsulated belief strength may account for the changes in post-recall affect and self characteristics. Participants reported higher state self-esteem and positive affect and lower negative affect in Session 3 compared to Session 1. This finding can be explained in the light of the existing explanations of the cognitive changes behind ImRS. In line with the retrieval competition hypothesis (Brewin, 2006) and with the SMS model (e.g. Conway et al., 2004), for example, it may be argued that participants were able to access a more positive working self after recalling the memory in the follow-up session as a result of ImRS. By modifying the meaning and valence of the adverse SDM, ImRS may have allowed participants to put their experience in the context of their current life and integrate it with the rest of their autobiographical memories. This may have reduced the salience of the memory-related working self and its retrieval advantage compared to positive working selves. It may be argued that ImRS made positive working selves more likely to be activated in the presence of cues (e.g. reminders) that would previously trigger the activation of working selves containing intrusive images related to the adverse memory.

The above explanation is based on the assumption of the retrieval competition hypothesis that negative working selves are permanently available. However, there is evidence (e.g. Kindt, Soeter, \& Vervliet, 2009; Schiller et al., 2010) that fear responses can be permanently eliminated under certain conditions (e.g. if the reconsolidation of the fear memories is disrupted or updated), suggesting a genuine modification of the fear-related structures. An alternative explanation of our findings which lends support to Arntz's (2011) hypothesis, therefore, is that ImRS changed the content of the memory-related working selves rather than their accessibility.

A final explanation for our findings is that ImRS may have modified the meaning participants had attributed to their memory and enabled them to express cognitions and emotions that they experienced but did not express during the adverse experience (see Dibbets \& Arntz, 2016). ImRS, therefore, may have had a primarily cathartic effect as a result of which participants reported increased positive affect and reduced negative affect following memory recall in Session 3. The more positive evaluations of the self may have been a result of the change in affect rather than of the ImRS modifying the accessibility or content of participants' working selves.

The study did not find significant changes in self-structure and post-recall state self-concept clarity and self-consistency following ImRS. Participant and study characteristics may account for this finding. First, the study was underpowered overall and this may have prevented us from observing an effect on these variables. Second, participants tended to have integrative self-structures which may have protected them from fluctuations in state self-concept clarity. Because their working selves contained both positive and negative attributes, participants may have perceived a continuation between their pre- and post-recall working selves. Third, a change in self-structure may have been unnecessary since, overall, participants already had an adaptive structure. Alternatively, changes in self-structure may require a longer treatment and/or follow-up. Finally, our operationalisation of the working self may have been inaccurate. It could be that shifting from one working self to another does not affect the extent to which individuals are clear about who they are. After all, the 
main aim of the SMS is to help individuals maintain a stable and consistent sense of self (see Conway, 2005).

The study needs to be seen in the light of some limitations. First, we had a small sample composed almost exclusively of females with a relatively low previous exposure to adverse experiences. In addition, we checked whether participants were receiving treatment for psychological issues at the time of data collection, but we did not screen them for current issues that may have been untreated. Although participants' adverse SDMs were similar to those of individuals suffering from psychological disorders and some participants may have been experiencing psychological issues at the time of data collection, their coping strategies may have been different from those of individuals experiencing significant distress. As a result, our findings may not generalise to the wider population or to clinical populations. Second, we did not have a control condition, so we cannot exclude the possibility that factors other than ImRS were responsible for the observed changes. As Çili and Stopa (2016) found, exposure alone may modify the impact of negative SDM recall on the working self. Finally, and perhaps more importantly, the methodology of the study does not allow us to draw any definitive conclusions on the mechanisms of change implicated in ImRS and the involvement of the self.

Despite these limitations, to the best of our knowledge this is the first study to investigate both cognitive and self changes after ImRS and to link social psychological theories and models of the self to the investigation of this technique. It suggests that ImRS helps individuals to update the meaning attached to adverse experiences and may facilitate the activation of more positive newly created or already existing self-representations in response to the retrieval of adverse memories, thus reducing the negative impact that these memories have on individuals' sense of self. An implication of these findings is that cognitive-behavioural therapists administering ImRS and other interventions targeting adverse memories may need to place greater emphasis on the relationship that these memories have with patients' sense of self. The outcomes of ImRS, including symptom alleviation and behavioural changes, may be a result of deeper cognitive changes in the way individuals perceive themselves. These changes may include - but not be limited to-maladaptive core beliefs, which may be just a part of the working selves that are associated with adverse memories. Clinicians, therefore, may need to focus on patients' broader sense of self rather than focusing primarily on core beliefs in order to enhance therapeutic outcomes.

Our findings are encouraging and present a potential explanation for why ImRS is effective by implicating the self. Clearly, more research is needed to replicate these findings and discard alternative explanations of the changes promoted by this technique. Future research can build on them by studying the self outcomes of ImRS in clinical populations; by including larger samples, control conditions, and longer follow-ups; and by developing better ways of assessing the self-images and goals making up the working self. In particular, we believe that future research would benefit from the development of more sensitive measures of self-images and the use of behavioural tasks in order to understand the different cognitions and goals that are activated immediately following memory recall. Ultimately, research focusing on the link that adverse memories have with patients' sense of self may enable us to understand the basic mechanisms operating in ImRS and to identify ways in which this therapeutic technique can be made more effective. 


\section{Acknowledgements}

The authors are grateful to Nicola Motton and Elaine Cockerham for their assistance during the study.

\section{Disclosure statement}

No potential conflict of interest was reported by the authors.

\section{Funding}

This study was conducted as part of the PhD research project of Soljana Çili, who was funded by the School of Psychology at the University of Southampton and by the Overseas Research Students Awards Scheme.

\section{ORCID}

Soljana Çili (iD) http://orcid.org/0000-0001-6137-0855

\section{References}

Arntz, A. (2011). Imagery rescripting for personality disorders. Cognitive and Behavioral Practice, 18, 466-481. http://dx.doi.org/10.1016/j.cbpra.2011.04.006

Arntz, A., Sofi, D., \& van Breukelen, G. (2013). Imagery Rescripting as treatment for complicated PTSD in refugees: A multiple baseline case series study. Behaviour Research and Therapy, 51, 274-283. http://dx.doi.org/10.1016/j.brat.2013.02.009

Arntz, A., \& Weertman, A. (1999). Treatment of childhood memories: Theory and practice. Behaviour Research and Therapy, 37, 715-740. http://dx.doi.org/10.1016/S0005-7967(98)00173-9

Beike, D. R., \& Wirth-Beaumont, E. T. (2005). Psychological closure as a memory phenomenon. Memory, 13, 574-593. http://dx.doi.org/10.1080/09658210444000241

Brewin, C. R. (2006). Understanding cognitive behaviour therapy: A retrieval competition account. Behaviour Research and Therapy, 44, 765-784. http://dx.doi.org/10.1016/j.brat.2006.02.005

Brewin, C. R., Gregory, J. D., Lipton, M., \& Burgess, N. (2010). Intrusive images in psychological disorders: Characteristics, neural mechanisms, and treatment implications. Psychological Review, 117, 210-232. http://dx.doi.org/10.1037/a0018113

Brewin, C. R., Wheatley, J., Patel, T., Fearon, P., Hackmann, A., Wells, A., ... Myers, S. (2009). Imagery rescripting as a brief stand-alone treatment for depressed patients with intrusive memories. Behaviour Research and Therapy, 47, 569-576. http://dx.doi.org/10.1016/j.brat.2009.03.008

Campbell, J. D., Trapnell, P. D., Heine, S. J., Katz, I. M., Lavallee, L. F., \& Lehman, D. R. (1996). Self-concept clarity: Measurement, personality correlates, and cultural boundaries. Journal of Personality and Social Psychology, 70, 141-156. http://dx.doi.org/10.1037/0022-3514.70.1.141

Ciili, S., \& Stopa, L. (2015). The retrieval of self-defining memories is associated with the activation of specific working selves. Memory, 23, 233-253. http://dx.doi.org/10.1080/09658211.2014.882955

Çili, S., \& Stopa, L. (2016). Impact of online exposure to adverse experiences on memory characteristics and activation of self-representations. Manuscript in preparation.

Constantino, M. J., Wilson, K. R., Horowitz, L. M., \& Pinel, E. C. (2006). The direct and stressbuffering effects of self-organization on psychological adjustment. Journal of Social and Clinical Psychology, 25, 333-360. http://dx.doi.org/10.1521/jscp.2006.25.3.333

Conway, M. A. (2005). Memory and the self. Journal of Memory and Language, 53, 594-628. http://dx.doi.org/10.1016/j.jml.2005.08.005

Conway, M. A., Meares, K., \& Standart, S. (2004). Images and goals. Memory, 12, 525-531. http://dx.doi.org/10.1080/09658210444000151 
Conway, M. A., \& Pleydell-Pearce, C. W. (2000). The construction of autobiographical memories in the self-memory system. Psychological Review, 107, 261-288. http://dx.doi.org/10.1037/ 0033-295X.107.2.261

Conway, M. A., Singer, J. A., \& Tagini, A. (2004). The self and autobiographical memory: Correspondence and coherence. Social Cognition, 22, 491-529. http://dx.doi.org/10.1521/soco.22.5.491.50768

Dibbets, P., \& Arntz, A. (2016). Imagery rescripting: Is incorporation of the most aversive scenes necessary? Memory, 24, 683-695. http://dx.doi.org/10.1080/09658211.2015.1043307

Dibbets, P., Poort, H., \& Arntz, A. (2012). Adding imagery rescripting during extinction leads to less ABA renewal. Journal of Behavior Therapy and Experimental Psychiatry, 43, 614-624. http:// dx.doi.org/10.1016/j.jbtep.2011.08.006

Emmons, R. A. (1986). Personal strivings: An approach to personality and subjective wellbeing. Journal of Personality and Social Psychology, 51, 1058-1068. http://dx.doi.org/10.1037/ 0022-3514.51.5.1058

Foa, E. B., Cashman, L., Jaycox, L., \& Perry, K. (1997). The validation of a self-report measure of posttraumatic stress disorder: The Posttraumatic Diagnostic Scale. Psychological Assessment, 9, 445-451. http://dx.doi.org/10.1037/1040-3590.9.4.445

Goodman, L. S., Corcoran, C., Turner, K., Yuan, N., \& Green, B. L. (1998). Assessing traumatic event exposure: General issues and preliminary findings for the Stressful Life Events Screening Questionnaire. Journal of Traumatic Stress, 11, 521-542.http://dx.doi.org/10.1023/A:1024456713321

Grunert, B. K., Smucker, M. R., Weis, J. M., \& Rusch, M. D. (2003). When prolonged exposure fails: Adding an imagery-based cognitive restructuring component in the treatment of industrial accident victims suffering from PTSD. Cognitive and Behavioral Practice, 10, 333-346. http:// dx.doi.org/10.1016/S1077-7229(03)80051-2

Hackmann, A., Clark, D. M., \& McManus, F. (2000). Recurrent images and early memories in social phobia. Behaviour Research and Therapy, 38, 601-610. http://dx.doi.org/10.1016/ S0005-7967(99)00161-8

Hinrichsen, H., Morrison, T., Waller, G., \& Schmidt, U. (2007). Triggers of self-induced vomiting in bulimic disorders: The roles of core beliefs and imagery. Journal of Cognitive Psychotherapy: An International Quarterly, 21, 261-272. http://dx.doi.org/10.1891/088983907781494528

Jobson, L., \& O'Kearney, R. (2008). Cultural differences in retrieval of self-defining memories. Journal of Cognitive Psychotherapy: An International Quarterly, 39, 75-80. http://dx.doi. org/10.1177/0022022107312073

Kindt, M., Soeter, M., \& Vervliet, B. (2009). Beyond extinction: Erasing human fear responses and preventing the return of fear. Nature Neuroscience, 12, 256-258. http://dx.doi.org/10.1038/nn.2271

Kuhn, M. H., \& McPartland, T. S. (1954). An empirical investigation of self-attitudes. American Sociological Review, 19, 68-76. http://dx.doi.org/10.2307/2088175

Lee, S. W., \& Kwon, J.-H. (2013). The efficacy of Imagery Rescripting (IR) for social phobia: A randomized controlled trial. Journal of Behavior Therapy and Experimental Psychiatry, 44, 351-360. http://dx.doi.org/10.1016/j.jbtep.2013.03.001

Linville, P. W. (1987). Self-complexity as a cognitive buffer against stress-related illness and depression. Journal of Personality and Social Psychology, 52, 663-676. http://dx.doi.org/10.1037/ 0022-3514.52.4.663

Markus, H. (1977). Self-schemata and processing information about the self. Journal of Personality and Social Psychology, 35, 63-78. http://dx.doi.org/10.1037/0022-3514.35.2.63

McFarland, C., \& Ross, M. (1982). Impact of causal attributions on affective reactions to success and failure. Journal of Personality and Social Psychology, 43, 937-946. http://dx.doi.org/10.1037/ 0022-3514.43.5.937

McKay, C. D., Singer, J. A., \& Conway, M. A. (in press). Psychological disorders and autobiographical memory: Examining memory specificity, affective content, and meaning-making. In A. Mishara, P. Corlett, P. Fletcher, \& M. Schwartz (Eds), Phenomenological neuropsychiatry: How patient experience bridges clinic with clinical neuroscience. New York, NY: Springer.

Nezlek, J. B., \& Plesko, R. M. (2001). Day-to-day relationships among self-concept clarity, self-esteem, daily events, and mood. Personality and Social Psychology Bulletin, 27, 201-211. http://dx.doi. org/10.1177/0146167201272006 
Nilsson, J.-E., Lundh, L.-G., \& Viborg, G. (2012). Imagery rescripting of early memories in social anxiety disorder: An experimental study. Behaviour Research and Therapy, 50, 387-392. http:// dx.doi.org/10.1016/j.brat.2012.03.004

Paradise, A. W., \& Kernis, M. H. (2002). Self-esteem and psychological well-being: Implications of fragile self-esteem. Journal of Social and Clinical Psychology, 21, 345-361. http://dx.doi.org/10.1521/ jscp.21.4.345.22598

Reimer, S. G., \& Moscovitch, D. A. (2015). The impact of imagery rescripting on memory appraisals and core beliefs in social anxiety disorder. Behaviour Research and Therapy, 75, 48-59. http:// dx.doi.org/10.1016/j.brat.2015.10.007

Schiller, D., Monfils, M.-H., Raio, C. M., Johnson, D. C., LeDoux, J. E., \& Phelps, E. A. (2010). Preventing the return of fear in humans using reconsolidation update mechanisms. Nature, 463, 49-53. http://dx.doi.org/10.1038/nature08637

Showers, C. (1992). Compartmentalization of positive and negative self-knowledge: Keeping bad apples out of the bunch. Journal of Personality and Social Psychology, 62, 1036-1049. http://dx.doi. org/10.1037/0022-3514.62.6.1036

Showers, C. J., Ditzfeld, C. P., \& Zeigler-Hill, V. (2015). Self-concept structure and the quality of selfknowledge. Journal of Personality, 83, 535-551. http://dx.doi.org/10.1111/jopy.12130

Showers, C. J., Limke, A., \& Zeigler-Hill, V. (2004). Self-structure and self-change: Applications to psychological treatment. Behavior Therapy, 35, 167-184. http://dx.doi.org/10.1016/ S0005-7894(04)80010-8

Singer, J. A. \& Salovey, P. (1993). The remembered self: Emotion and memory in personality. New York, NY: Free Press.

Smucker, M. R., Dancu, C., Foa, E. B., \& Niederee, J. L. (1995). Imagery rescripting: A new treatment for survivors of childhood sexual abuse suffering from posttraumatic stress. Journal of Cognitive Psychotherapy: An International Quarterly, 9, 3-17.

Speckens, A. E. M., Hackmann, A., Ehlers, A., \& Cuthbert, B. (2007). Intrusive images and memories of earlier adverse events in patients with obsessive compulsive disorder. Journal of Behavior Therapy and Experimental Psychiatry, 38, 411-422. http://dx.doi.org/10.1016/j.jbtep.2007.09.004

Spielberger, C. D., Gorsuch, R. L., Lushene, R., Vagg, P. R., \& Jacobs, G. A. (1983). Manual for the state-trait anxiety inventory (Form Y). Palo Alto, CA: Mind Garden.

Sutherland, K., \& Bryant, R. A. (2005). Self-defining memories in post-traumatic stress disorder. British Journal of Clinical Psychology, 44, 591-598. http://dx.doi.org/10.1348/014466505X64081

Watson, D., Clark, L. A., \& Tellegen, A. (1988). Development and validation of brief measures of positive and negative affect: The PANAS scales. Journal of Personality and Social Psychology, 54, 1063-1070. http://dx.doi.org/10.1037/0022-3514.54.6.1063

Wells, A., \& Hackmann, A. (1993). Imagery and core beliefs in health anxiety: Content and origins. Behavioural and Cognitive Psychotherapy, 21, 265-273. http://dx.doi.org/10.1017/ S1352465800010511

Wild, J., Hackmann, A., \& Clark, D. M. (2007). When the present visits the past: Updating traumatic memories in social phobia. Journal of Behavior Therapy and Experimental Psychiatry, 38, 386-401. http://dx.doi.org/10.1016/j.jbtep.2007.07.003

Wild, J., Hackmann, A., \& Clark, D. M. (2008). Rescripting early memories linked to negative images in social phobia: A pilot study. Behavior Therapy, 39, 47-56. http://dx.doi.org/10.1016/j. beth.2007.04.003

Young, J. E. (1999). Cognitive therapy for personality disorders: A schema-focused approach (3rd ed.). Sarasota, FL: Professional Resource Press.

Young, J. E., Klosko, J. S., \& Weishaar, M. E. (2003). Schema therapy: A practitioner's guide. New York, NY: Guilford Press.

Zeigler-Hill, V., \& Showers, C. J. (2007). Self-structure and self-esteem stability: The hidden vulnerability of compartmentalisation. Personality and Social Psychology Bulletin, 33, 143-159. http://dx.doi.org/10.1177/0146167206294872 